\title{
Phytoprostanes and phytofurans -oxidative stress and bioactive compounds- in almonds are affected by deficit irrigation in almond trees
}

Leontina Lipan ${ }^{1}$, Jacinta Collado-González ${ }^{2}$, Raúl Domínguez-Perles ${ }^{2 *}$, Mireia Corell ${ }^{3,4}$, Valérie Bultel-Poncé 5 , Jean-Marie Galano ${ }^{5}$, Thierry Durand ${ }^{5}$, Sonia Medina ${ }^{2}$; Ángel Gil-Izquierdo ${ }^{2 *}$, Ángel Carbonell-Barrachina ${ }^{1}$

${ }^{1}$ Universidad Miguel Hernández de Elche (UMH), Escuela Politécnica Superior de Orihuela (EPSO), Department of Agro-Food Technology, Research Group "Food Quality and Safety", Carretera de Beniel, km 3.2, 03312-Orihuela, Alicante, Spain.

${ }^{2}$ Research Group on Quality, Safety and Bioactivity of Plant Foods, Departamento de Ciencia y Tecnología de Alimentos (CEBAS-CSIC), Campus de Espinardo-25, 30100, Murcia, Spain.

${ }^{3}$ Dpto. Ciencias Agroforestales, Escuela Técnica Superior de Ingeniería Agronómica, Universty of Sevilla, Ctra. de Utrera, km 1, 41013, Sevilla, Spain.

${ }^{4}$ Associated Unity to CSIC: Uso Sostenible del Suelo y el Agua en la Agricultura (Universidad de Sevilla-Instituto de Recursos Naturales y Agrobiología de Sevilla), Ctra. de Utrera km 1, 41013 Sevilla, Spain.

${ }^{5}$ Institut des Biomolécules Max Mousseron (IBMM), UMR 5247, CNRS, University of Montpellier, ENSCM, Faculty of Pharmacy, Montpellier, France.

* Correspondence: rdperles@cebas.csic.es; Tel.: +34 968396200 Ext. 6247; angelgil@,cebas.csic.es; Tel.: +34968396200 Ext. 6363 

Supplemental Table 1. UHPLC/MS/MS parameters for the quantification and confirmation of PhytoPs and PhytoFs in almonds of "Vairo" cultivar under water stress.

\begin{tabular}{|c|c|c|}
\hline Compound & Retention time (min) & MRM transition $(\mathrm{m} / \mathrm{z})$ \\
\hline \multicolumn{3}{|l|}{ Phytoprostanes } \\
\hline ent-16-epi-16- $\mathrm{F}_{1 \mathrm{t}}-\mathrm{PhytoP}$ & 1.807 & $327.1>283.2$ \\
\hline ent- $16-\mathrm{F}_{1 \mathrm{t}}-\mathrm{PhytoP}$ & 1.807 & $327.1>283.2$ \\
\hline $9-F_{1 t}-$ PhytoP & 1.913 & $327.2>171.2$ \\
\hline 9-epi-9-F $F_{1 \mathrm{t}}-\mathrm{PhytoP}$ & 2.132 & $327.2>171.2$ \\
\hline ent-9-epi-9-D ${ }_{1 t}-$ PhytoP & 2.133 & $325.2>307.2$ \\
\hline ent-9-D $\mathrm{D}_{1 \mathrm{t}}$-PhytoP & 2.491 & $325.2>307.2$ \\
\hline 16-B -PhytoP $^{-}$ & 3.230 & $307.2>235.2$ \\
\hline 9- $\mathrm{L}_{1}-\mathrm{PhytoP}$ & 3.507 & $307.2>185.1$ \\
\hline \multicolumn{3}{|l|}{ Phytofurans } \\
\hline ent-9(RS)-12-epi-ST- $\Delta^{10}-13-P h y t o F$ & 1.650 & $344.0>300.0$ \\
\hline ent-16(RS)-9-epi-ST- $\Delta^{14}-10-$ PhytoF & 1.667 & $343.9>201.1$ \\
\hline ent-16(RS)-13-epi-ST- $\Delta^{14}-9$-PhytoF & 1692 & $343.0>171.1$ \\
\hline
\end{tabular}

